\title{
Disease in Severe Mental Illness
}

\author{
Dinesh Bhugra
}

Accepted: 30 April 2014/Published online: 17 May 2014

(C) Springer India Pvt. Ltd. 2014

The distinction between disease and illness is critical in psychiatric disorders. There is no doubt that cultures define deviance and abnormality especially when it is related to behaviour. For psychiatric and other mental health professionals, it is important that the focus of their therapeutic alliance and therapeutic endeavours remains the patient.

Kleinman et al. [1] describe disease as abnormalities in the structure and function of body organs and systems, whereas patients suffer from illnesses (which are experiences of disvalued changes in the term of being and social function). Others have made similar observations [2-4]. Illness may occur in the absence of disease. In the Indian context, conversion disorders may fall into this category. Disease may also be seen in the context of Western paradigms of medicine whereas illness is something folk healers may be better at dealing with. It is entirely possible that experiences and descriptions of illness are all embedded in the social, family and cultural nexus [5].

Illness is culturally shaped as cultures determine explanations of what affects us, the representations of these experiences and idioms used to express our distress. Physicians identify, diagnose and manage diseases, as that is what they are trained in, whereas patients are more interested in social functioning. This becomes even more important and relevant in chronic severe mental or physical illness where patients may be able to live with their symptoms provided they can hold down jobs, have money, social support and friends they are able to rely on.

Disease can also refer to a described and recognisable combination of symptoms and signs or phenomena

D. Bhugra $(\square)$

Health Service \& Population Research Department, Institute of Psychiatry, King's College London, PO25, London, UK

e-mail: dinesh.bhugra@kcl.ac.uk associated with specified disorders of structure or function or phenomena due to a specific cause - whether these are single or multiple [6]. Campbell et al. [6] go on to suggest that disease is the sum of the abnormal phenomena displayed by a group of living organisms in association with a specified common characteristic or set of characteristics by which they differ from the norm for that species in such a way so as to place them at a biological disadvantage! They report that most people without medical training see disease as an agent causing illness but, as pointed out earlier, illnesses can exist without any evidence of disease. They make an interesting point that within the therapeutic encounter both doctor and patient may agree on 'disease' so as to find and deal with a so-called common enemy. However, when medical students are asked to define 'disease', not surprisingly their observations fall somewhere in between the lay and the professional models [7, 8]. When Helman [9] looked at the models of disease and illness used in general practice he argued that folk theories of causes of ill health are many and patients may treat themselves or under advice from friends and relatives before and during their contact with formal health sector.

However, when applied to chronic severe mental illness, these models have to be carefully reviewed not only in the cultural context but also within polysystems approach. Depending upon explanatory models being used by the patients and their carers, different pathways into 'professional' care may emerge. Often in many countries patients will use a combination of approaches. For example, in India patients will often take medication prescribed by allopathic practitioners along with homeopathic or Ayurvedic medication. The explanatory models may be seen as genetic, biological, social or occasionally psychological. The perceived locus of control will also determine where the first port of call for help will lie. For example, if the 
locus of control is seen as sins related to past birth or neighbour's evil eye, then the first stop for care may well be with shamans or religious healers. Helman [9] suggests that the four common categories of causation within folk models of illness will include invasion, degeneration, mechanical or balance as described by Chrisman [10] are not entirely discrete and tend to overlap.

This distinction between disease and illness remains important in making sense of the patient experience within the therapeutic encounter. Many patients with chronic severe mental illness can manage to cope with their psychotic symptoms provided they are functioning reasonably well in their social or personal spheres. This allows them to manage their 'illness' in the presence of disease. The challenge for clinicians is to feel confident and comfortable to ignore the option that they may not be able to eradicate the symptoms. Such an approach also leads to specific issues in setting up epidemiological research and studies to explore prevalence of conditions. A straightforward simple approach to usual or standard epidemiology which looks at single causative factors thus may not be entirely applicable.

The health care systems have their own cultures in understanding or expanding the diagnosis, patient experience and management. As Fox [11] points out so called over-medicalisation of American society refers simply to an increase in kinds of attitudes and behaviours that have come to be defined as illness and subjected to medical jurisdiction. This does not take into account earlier assertions in the society where many of these attitudes and behaviours were seen as sinful. Fox also argues that in a more secular but less scientifically and medically oriented society, many of these attitudes may be seen as criminal and therefore dealt within the judicial system. This observation is important as this reflects the continuing process of divestment away from sin and crime as categories and moving towards illnesses as explanatory concepts. This may lead to an expansion in the number of illnesses [12]. However, does this also mean that the number of diseases will rise? Another additional issue which deserves further exploration and discussion is the role laboratory investigations play in increasing 'pathology' or 'disease' but not leading to illness. It has been estimated that as much as $90 \%$ of apparently healthy individuals have some physical aberration [13]. Fox [11] asks an important question: the extent to which illness is an objective reality, subjective state or a special construct? The role of biotechnological advances in creating more diseases also needs discussion and perhaps challenging. Does an increased number of diagnostic categories lead to better management of illnesses caused by these diagnoses?

The social, political and economic resources dedicated to illness do not always take mental illness by itself or as a consequence of physical illness into account. This is the challenge that psychiatrists need to address as a matter of urgency especially when attempting to manage individuals with chronic severe mental illness. A clearer understanding of the definitions and characteristics of disease and its conversion to illness and resulting impact on the individual must form the starting point when setting up services and delivering what is needed.

\section{References}

1. Kleinman A, Eisenberg L, Good B. Culture, illness and care: clinical lessons from anthropological and cross-cultural research. Focus. 2006;IV:140-9.

2. Eisenberg L. Disease and illness: distinction between professional and popular ideas of sickness. Cult Med Psychiatry. 1977;1:9-23.

3. Feinstein AP. Clinical judgement. Baltimore: Williams and Wilkins; 1967. p. 24-5.

4. Engel GL. The ned for a new model: a challenge for biomedicine. Science. 1977;196:129-36.

5. Litman TJ. The family as a basic unit in health and medical care. Soc Sci Med. 1974;8:495-519.

6. Campbell EJM, Scadding JG, Roberts RS. The concept of disease. BMJ. 1979;2:757-62.

7. Erueti C, Glasziou P, Mar CD, van Driel ML. Do you think it's a disease? A survey of medical students. BMC Med Educ. 2012;12:19-22.

8. Stefan MD, McManus IC. The concept of disease: its evolution in medical students. Soc Sci Med. 1989;29:791-2.

9. Helman C. Disease versus illness in general practice. J RCGP. 1981;31:548-52.

10. Chrisman NJ. The health seeking process: an approach in the natural history of illness. Cult Med Psych. 1977;1:351-77.

11. Fox RC. The medicalization and demedicalization of American society. Daedalus. 1977;106:9-22.

12. Sedgewick P. Illness, mental and otherwise: all illnesses express a social judgment. Hastings Cent Stud. 1973;1(3):19-40.

13. Zola IK. Culture and symptoms-an analysis of patients' presenting complaints. Am Soc Rev. 1966;31:615-30. 